\title{
The evolution of probability functions in an inelasticly deforming two-phase medium
}

\author{
Brent L. Adams \\ b_I_adams@byu.edu \\ H. Garmestani
}

S. Lin

Follow this and additional works at: https://scholarsarchive.byu.edu/facpub

Part of the Mechanical Engineering Commons

Original Publication Citation

International Journal of Solids and Structures 37 (2) 423-434

\section{BYU ScholarsArchive Citation}

Adams, Brent L.; Garmestani, H.; and Lin, S., "The evolution of probability functions in an inelasticly deforming two-phase medium" (1998). Faculty Publications. 632.

https://scholarsarchive.byu.edu/facpub/632

This Peer-Reviewed Article is brought to you for free and open access by BYU ScholarsArchive. It has been accepted for inclusion in Faculty Publications by an authorized administrator of BYU ScholarsArchive. For more information, please contact ellen_amatangelo@byu.edu. 


\title{
The evolution of probability functions in an inelasticly deforming two-phase medium
}

\author{
S. Lin ${ }^{a}$, H. Garmestani ${ }^{a}$,, B. Adams ${ }^{\mathrm{b}}$ \\ ${ }^{a}$ Department of Mechanical Engineering at FAMU-FSU College of Engineering, and Center for Materials Research and Technology \\ (MARTECH), Tallahassee, FL 32310-6046, USA \\ ${ }^{\mathrm{b}}$ Carnegie Mellon University, Pittsburg, PA 15213-3890, USA
}

\begin{abstract}
A formulation is introduced here for the evolution of correlation functions in an inelastically deforming two phase medium. Probability functions play a major role in describing the statistical distribution of different phases in a heterogeneous medium in the development of statistical continuum theory. Proper formulation of statistical continuum model for inelastic deformation requires better understanding of the evolution of the corresponding probability functions. A two point probability function representation is used to approximate the statistical correlation functions. The evolution of these functions requires the information from higher order probability functions, in this case, a three point probability function. A decomposition of this three point probability function is required for the simulation of the statistical model. The results were compared with experimental data.
\end{abstract}

\section{Introduction}

Statistical continuum theory for the treatment of the effective elastic properties of materials was initiated independently by Volkov and Klinskikh (1965), Lomakin (1965), Beran and Molyneux (1966) and Kröner (1967). Later, Beran (1968), Lomakin (1970), Kröner (1972, 1977, 1987) and McCoy (1981) extended this theory to obtain better approximations to the effective properties of random microstructures. The application of the statistical theory has found more interest in the prediction of large deformation of polycrystalline metals as compared with composite materials. Such efforts provided an alternative to other popular methodologies, like the self-consistent model (Molinari et al., 1987).

Statistical continuum theories are based on proper representation of statistical distribution functions.

\footnotetext{
* Corresponding author. Tel.: + 1-850-644-5993; Fax: + 1-850-644-9281.

E-mail address: garm@magnet.fsu.edu (H. Garmestani)
} 
Most of the work in this area concentrated on the incorporation of probability functions into elastic and crystal plasticity problems (Kröner, 1987). The properties of the probability functions themselves and their proper formulation in this area has received little attention in the past (Adams, 1986; Wang et al., 1990). A theory for statistical mechanics analysis of viscoplastic behavior in heterogeneous polycrystals has been presented by Adams et al. (1989). The formulation used the two Green's function solution presented by Molinari et al. (1987) to formulate the local correlation functions. A methodology for the evolution of the probability functions was also proposed in that work. Recently, a simplified statistical model was presented by Garmestani et al. (1998) to apply the statistical model to an inelastically deforming composite structure. In that work the evolution of the microstructure was totally ignored and it was further assumed that the probability functions remain constant during deformation. Similar to the previous work, two point probability function are used to represent the heterogeneity. In the present paper the evolution of the probability functions is investigated in detail. The understanding of this process is important in the predication of mechanical properties of a heterogeneous medium especially for large deformation processes. Different well known examples of a two-phase composite material are chosen in the present work and their probability functions are characterized. The two phases chosen resemble a hard-soft composite. The second phase (fiber, reinforcement,...) distribution and morphology is represented by the two point probability functions. The strain rate is taken to be proportional to the resolved local shear stress raised to a power which is different for the two constituents. Contributions to the strain rate due to elasticity are neglected. A two-Green's function solution to the equations of stress equilibrium, originally proposed by Molinari et al. (1987), is utilized to obtain the constitutive law for the heterogeneous medium. This relation links the local velocity gradient to the fields of the local viscoplastic modulus and macroscopic velocity gradient. The concepts of statistical continuum theory are introduced into the localization relation to obtain a closed form solution. The resulting model calculates an effective modulus for the two-phase composite medium.

Analytical forms for the two-point and three-point probability functions to describe the distribution and morphology of a two-phase composite material have been presented earlier by Corson (1974). In that work, an exponential function has been used for the two-point and higher order probability functions. Such a formulation assumes that the probability function is independent of orientation which is not necessarily correct. The orientational dependence can be incorporated by generating the probability functions for some important orientations. The generalized form for the two point probability function, based on spherical harmonics, was introduced earlier by Adams et al. (1987). The use of such a general function may not be necessary in most cases and simpler forms, based on an extension of the form used in this paper may be adequate. The use of a closed form formulation is necessary for faster simulation of the evolution process.

A methodology based on the divergence theorem originally proposed by Adams et al. (1987) is used here to formulate the evolution of the two-point probability function. The numerical simulations are compared to experimental results to investigate the validity of the model. An aluminum based composite material reinforced with $\mathrm{SiC}$ particulates was chosen here to acquire the experimental data. Several composite specimens were deformed inelastically to different strain levels. The specimens were then examined and the correlation functions were extracted digitally. This information was then compared to the theoretical simulations using the statistical model.

\section{Statistical formulation and probability functions}

The nonlinear constitutive law for the viscoplastic behavior of individual constituents in the composite may be expressed as 


$$
D_{i j}=M_{i j k l}(\mathbf{S}, h) S_{k l},
$$

where $D_{i j}$ is strain-rate, $S_{k l}$ is the deviatoric Cauchy stress and $M_{i j k l}$ denotes the instantaneous compliance tensor which depends upon the stress and the local state variables denoted by $h$. Because the composite only includes two phases, $h$ has two values associated with the two phases. The inverse relationship for stress and strain-rate is given as

$$
S_{i j}=N_{i j k l}(\mathbf{D}, h) D_{k l}=N_{i j k l}(\mathbf{D}, h) v_{k, l},
$$

where, $\mathbf{N}=\mathbf{M}^{-1}, v_{k, l}$ is the velocity gradient tensor.

The elastic strain is neglected, and incompressibility is imposed globally to give $v_{i, i}=0$. Combining this condition with the equilibrium equations $\left(\sigma_{i j, j}=0\right)$ of the local Cauchy stress $\left(\sigma_{i j}=S_{i j}-p \delta_{i j}\right.$, where $p$ is the hydrostatic pressure), a simultaneous set of partial differential equations containing four equations and four unknowns (three $v$ 's and one $p$ ) is obtained. Assuming that the medium is unbounded and subject to a uniform macroscopic velocity gradient $\bar{v}_{i, j}$, the Green's function method can be used to solve this set of equations. The local velocity gradient can then be expressed as

$$
L_{i k}(\mathbf{r})=\bar{L}_{i k}+\int_{\mathbf{r}^{\prime} \in V} G_{i j, k l}\left(\mathbf{r}-\mathbf{r}^{\prime}\right) \tilde{N}_{j l r s}\left(\mathbf{L}\left(\mathbf{r}^{\prime}\right), h\left(\mathbf{r}^{\prime}\right)\right) L_{r s}\left(\mathbf{r}^{\prime}\right) \mathrm{d} \mathbf{r}^{\prime},
$$

where $L_{i k}=v_{i, k}, \mathbf{G}$ is the Green function that is calculated using the average moduli $\int \mathbf{N}(\overline{\mathbf{L}}, h) f(h) \mathrm{d} h, \tilde{\mathbf{N}}$ is the so-called polarized modulus which is defined by the following:

$$
\tilde{N}_{i j k l}=N_{i j k l}-\int N_{i j k l}(\overline{\mathbf{L}}, h) f(h) \mathrm{d} h,
$$

where $h$ represents the local state variable which determines the secant moduli, $f(h)$ is the one-point probability density for the occurrence of state $h$ in the medium.

In short notation, Eq. (3) is written as $\mathbf{L}=\overline{\mathbf{L}}+\mathbf{G} * \tilde{\mathbf{N}} \mathbf{L}$. Assume that local velocity gradients are not far from the macroscopic velocity gradient: $\left|\left(v_{i, j}-\bar{v}_{i, j}\right) / \bar{v}_{i, j}\right|<1$ for all $i, j$. Then Eq. (3) can be expressed in a Taylor series expansion about $\overline{\mathbf{L}}$ as

$$
\mathbf{L}=\overline{\mathbf{L}}+\mathbf{G} *\left((\tilde{\mathbf{N}} \overline{\mathbf{L}})+\left.\partial(\tilde{\mathbf{N}} \mathbf{L})\right|_{\mathbf{L}=\overline{\mathbf{L}}}(\mathbf{L}-\overline{\mathbf{L}})+\left.\frac{1}{2 !} \partial^{2}(\tilde{\mathbf{N}} \mathbf{L})\right|_{\mathbf{L}=\overline{\mathbf{L}}}(\mathbf{L}-\overline{\mathbf{L}})^{2}+\ldots\right),
$$

where

$$
\left.\partial(\tilde{\mathbf{N}} \mathbf{L})\right|_{\mathbf{L}=\overline{\mathbf{L}}}=\left.\frac{\partial\left(\tilde{N}_{i j k l} L_{k l}\right)}{\partial L_{r s}}\right|_{\mathbf{L}=\overline{\mathbf{L}}} \text { and }\left.\quad \partial^{2}(\tilde{\mathbf{N}} \mathbf{L})\right|_{\mathbf{L}=\overline{\mathbf{L}}}=\left.\frac{\partial^{2}\left(\tilde{N}_{i j k l} L_{k l}\right)}{\partial L_{r s} \partial L_{t u}}\right|_{\mathbf{L}=\overline{\mathbf{L}}} .
$$

In the following, the concept of statistical continuum mechanics will be introduced through the ensemble averaging of the quantities defined in the equations above. Such averaging processes require the introduction of two higher order probability functions for the first and higher order corrections. It will be shown that the calculation of the first order correction requires the use of two point correlation functions, whereas the second order correction requires the information on the three point probability functions. This paper is primarily focused on the contribution of the two point correlation functions in the evolution of heterogeneous microstructures. In the final analysis, the second order correction was calculated using an iterative method and was found to be negligible compared to the first-order correction for the microstructures chosen in this study. As a result, we focus our attention to the first-order correction to the homogeneous Taylor's assumption (Taylor, 1938). Then, Eq. (5) becomes: 


$$
\mathbf{L}=\overline{\mathbf{L}}+\mathbf{G} * \tilde{\mathbf{N}} \mathbf{L}
$$

Taking the ensemble average of the two sides of Eq. (6):

$$
\langle\mathbf{L}(\mathbf{r})\rangle_{h_{n}}=\overline{\mathbf{L}}+\mathbf{G}\left(\mathbf{r}-\mathbf{r}^{\prime}\right) *\left\langle\tilde{\mathbf{N}}\left(\overline{\mathbf{L}}, h\left(\mathbf{r}^{\prime}\right)\right)\right\rangle_{h_{n}} \overline{\mathbf{L}} .
$$

Here, $\langle\mathbf{L}(\mathbf{r})\rangle_{h_{n}}$ prescribes the partial ensemble average of the local velocity gradient in particles of state $h_{n}$. In other words, $\left\langle\tilde{\mathbf{N}}\left(\mathbf{L}, h\left(\mathbf{r}^{\prime}\right)\right)\right\rangle_{h_{n}}$ is the ensemble average of the polarized modulus in particles at $\mathbf{r}^{\prime}$, given $\mathbf{r} \in h_{n}$, and the subscript $n=1,2$ for the two phases. In this paper, $h$ has two values: $h_{1}$ and $h_{2}$, representing phase 1 and phase 2 in the medium, respectively; then:

$$
\begin{aligned}
& \langle\tilde{\mathbf{N}}(\mathbf{L}, h)\rangle_{h_{1}}=f\left(\mathbf{r}^{\prime} \in h_{1} \mid \mathbf{r} \in h_{1}\right) \tilde{\mathbf{N}}^{1}(\overline{\mathbf{L}})+f\left(\mathbf{r}^{\prime} \in h_{2} \mid \mathbf{r} \in h_{1}\right) \tilde{\mathbf{N}}^{2}(\overline{\mathbf{L}}) \\
& \langle\tilde{\mathbf{N}}(\mathbf{L}, h)\rangle_{h_{2}}=f\left(\mathbf{r}^{\prime} \in h_{1} \mid \mathbf{r} \in h_{2}\right) \tilde{\mathbf{N}}^{1}(\overline{\mathbf{L}})+f\left(\mathbf{r}^{\prime} \in h_{2} \mid \mathbf{r} \in h_{2}\right) \tilde{\mathbf{N}}^{2}(\overline{\mathbf{L}}) .
\end{aligned}
$$

In general, $\left\langle\tilde{\mathbf{N}}\left(\overline{\mathbf{L}}, h\left(\mathbf{r}^{\prime}\right)\right)\right\rangle_{h_{n}}$ can be described in terms of the conditional 2-point probability function of state $h$ as

$$
\left\langle\tilde{\mathbf{N}}\left(\overline{\mathbf{L}}, h\left(\mathbf{r}^{\prime}\right)\right)\right\rangle_{h_{n}}=\int f\left(\mathbf{r}^{\prime} \in h \mid \mathbf{r} \in h_{n}\right) \tilde{\mathbf{N}}(\overline{\mathbf{L}}, h) \mathrm{d} h,
$$

where $f\left(\mathbf{r}^{\prime} \in h \mid \mathbf{r} \in h_{n}\right)$ is the probability that $\mathbf{r}^{\prime}$ belongs to state $h$ given $\mathbf{r}$ is at state $h_{n}$. It is easy to note that the solution to this problem lies in the existence of the proper probability functions for a specific medium. For the two-isotropic-phase medium used in this paper, Corson's (Corson, 1974) exponential form is assumed to be sufficient to represent the correlation functions.

$$
P_{i j}(r)=\alpha_{i j}+\beta_{i j} \exp \left(-c_{i j} r^{n_{i j}}\right),
$$

$i=1,2, j=1,2 . P_{i j}(r)$ is defined as the probability for the occurrence of one point in phase $i$ and the other point (which is located at a distance, $r$, away) in phase $j$. It is easy to see that the equation is isotropic or $P_{i j}$ is only a function of the magnitude of $\mathbf{r}-\mathbf{r}^{\prime}$, as shown in Eq. (7). The statistical parameters, $\alpha_{i j}$ and $\beta_{i j}$, are material constants that can be found from the limiting conditions of the variable $r$. These limiting conditions refer to $r$ equal to zero and infinity and that reduces the two point probability functions to one point probability functions or volume fractions $V_{1}$ and $V_{2}$ for the two phases (Table 1). These two constants do not change during deformation. The parameters, $c_{i j}$ and $n_{i j}$, however, depend on the microstructure distribution and will change during the evolution process.

The two constants, $c_{i j}$ and $n_{i j}$ are empirically determined by a least mean square fit to the measured probability data in Eq. (11). These material constants are calculated for specific microstructures and only change during evolution (deformation). The numerical routine involves the calculation of the probability functions $P_{i j}(r)$ for the initial microstructure using the procedure explained in this paper. These probability functions are calculated in terms of the distance, $r$. In the procedure which follows, it will be shown that what evolves during the numerical routines are the microstructures that are stored in the form of probability functions. The evolution is then defined in terms of changes in $r$ and, for each $r$, there is a corresponding probability function. In the following, the evolution of any length vector is described using the three point probability functions. The numerical fit is then performed by finding the corresponding materials constants, $c_{i j}$ and $n_{i j}$, for this change by keeping the probabilities the same for the final values of $r$.

The relation between $f$, a conditional probability, and $P_{i j}$, absolute probability, is shown in the following equations: 
Table 1

Limiting conditions on two-point probability functions

\begin{tabular}{|c|c|c|c|c|}
\hline \multirow[b]{2}{*}{$P_{i j}$} & \multicolumn{2}{|c|}{ Boundary conditions } & \multicolumn{2}{|c|}{ Resultant coefficients } \\
\hline & $r=0$ & $r \rightarrow \infty$ & $\alpha_{i j}=$ & $\beta_{i j}=$ \\
\hline$P_{11}$ & $V_{1}$ & $V_{1}^{2}$ & $V_{1}^{2}$ & $V_{1} V_{2}$ \\
\hline$P_{12}$ & 0 & $V_{1} V_{2}$ & $V_{1} V_{2}$ & $-V_{1} V_{2}$ \\
\hline$P_{21}$ & 0 & $V_{1} V_{2}$ & $V_{1} V_{2}$ & $-V_{1} V_{2}$ \\
\hline$P_{22}$ & $V_{2}$ & $V_{2}^{2}$ & $V_{2}^{2}$ & $V_{1} V_{2}$ \\
\hline
\end{tabular}

$$
\begin{aligned}
& f\left(\mathbf{r}^{\prime} \in h_{1} \mid \mathbf{r} \in h_{1}\right)=\frac{P_{11}}{V_{1}}, \\
& f\left(\mathbf{r}^{\prime} \in h_{2} \mid \mathbf{r} \in h_{1}\right)=\frac{P_{12}}{V_{1}}, \\
& f\left(\mathbf{r}^{\prime} \in h_{1} \mid \mathbf{r} \in h_{2}\right)=\frac{P_{21}}{V_{3}} \\
& f\left(\mathbf{r}^{\prime} \in h_{2} \mid \mathbf{r} \in h_{2}\right)=\frac{P_{22}}{V_{2}} .
\end{aligned}
$$

Table 1 shows the limiting conditions on the two-point probability functions.

\section{The evolution of two-point probability functions}

The probability functions represent the distribution and morphology of the microstructure in a heterogeneous material (composites) in a statistical continuum mechanics scheme. As the microstructure deforms, the probability functions are expected to evolve and, for a composite material, the evolution is incorporated in the changes with shape and distribution of the second phase.

Referring to Fig. 1, vector e originates from state $h$ and terminates at state $h^{\prime}$. The rate of change of

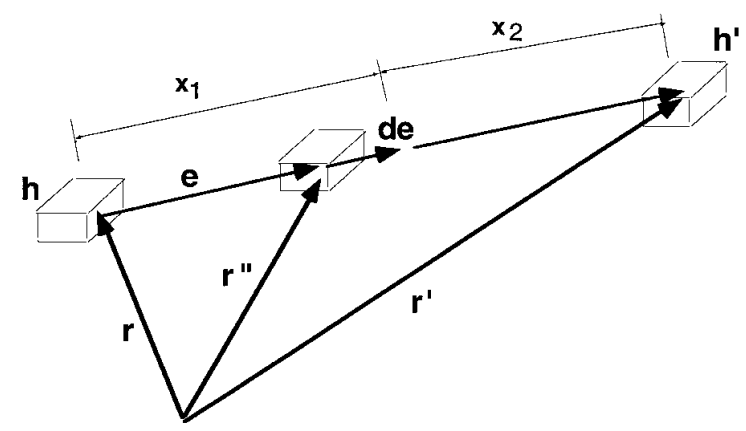

Fig. 1. Definition of parameters associated with the evolution equations. 
the magnitude of $\mathbf{e}$ can be written as

$$
\langle\dot{\mathbf{e}}\rangle_{h, h^{\prime}}=\int\left\langle\mathbf{L}\left(\mathbf{r}^{\prime \prime}\right)\right\rangle_{h, h^{\prime}} \cdot \mathrm{de},
$$

where $\left\langle\mathbf{L}\left(\mathbf{r}^{\prime \prime}\right)\right\rangle_{h, h^{\prime}}$ is the local strain rate represented by vector $\mathbf{r}^{\prime \prime}$ when the point represented by vector $\mathbf{r}$ which belongs to state $h$ and the point represented by vector $\mathbf{r}^{\prime}$ belongs to state $h^{\prime}$. Consequently, a three-point probability function is needed to obtain the value for $\left\langle\mathbf{L}\left(\mathbf{r}^{\prime \prime}\right)\right\rangle_{h, h^{\prime}}$ from $\left\langle\mathbf{L}\left(\mathbf{r}^{\prime \prime}\right)\right\rangle_{h^{\prime \prime}}$, which is derived from the two-point probability functions, as shown in the following.

$$
\begin{aligned}
\left\langle\mathbf{L}\left(\mathbf{r}^{\prime \prime}\right)\right\rangle_{h, h^{\prime}} & =\int f_{3}\left(h^{\prime \prime}, \mathbf{r}^{\prime \prime} \mid h^{\prime}, \mathbf{r}^{\prime}, h, \mathbf{r}\right)\left\langle\mathbf{L}\left(\mathbf{r}^{\prime \prime}\right)\right\rangle_{h^{\prime \prime}} \mathrm{d} h^{\prime \prime} \\
& =f_{3}\left(h_{1}, \mathbf{r}^{\prime \prime} \mid h^{\prime}, \mathbf{r}^{\prime}, h, \mathbf{r}\right)\left\langle\mathbf{L}\left(\mathbf{r}^{\prime \prime}\right)\right\rangle_{h_{1}}+f_{3}\left(h_{2}, \mathbf{r}^{\prime \prime} \mid h^{\prime}, \mathbf{r}^{\prime}, h, \mathbf{r}\right)\left\langle\mathbf{L}\left(\mathbf{r}^{\prime \prime}\right)\right\rangle_{h_{2}} .
\end{aligned}
$$

Here, $f_{3}\left(h^{\prime \prime}, \mathbf{r}^{\prime \prime} \mid h^{\prime}, \mathbf{r}^{\prime}, h, \mathbf{r}\right)$ is a three-point probability function and corresponds to the probability of occurrence of state $h^{\prime \prime}$ at $\mathbf{r}^{\prime \prime}$, given that state $h$ and $h^{\prime}$ occur at $\mathbf{r}$ and $\mathbf{r}^{\prime}$, respectively. $\left\langle\mathbf{L}\left(\mathbf{r}^{\prime \prime}\right)\right\rangle_{h_{1}}$ and $\left\langle\mathbf{L}\left(\mathbf{r}^{\prime \prime}\right)\right\rangle_{h_{2}}$ indicate the ensemble averages of the strain rates of state $h_{1}$ and $h_{2}$, respectively.

An approximation for the decomposition of the three-point probability function using the two-point probability functions is introduced here as

$$
f_{3}\left(h^{\prime \prime}, \mathbf{r}^{\prime \prime} \mid h^{\prime}, \mathbf{r}^{\prime}, h, \mathbf{r}\right) \cong \frac{x_{1}}{x_{1}+x_{2}} f_{2}\left(h^{\prime \prime}, \mathbf{r}^{\prime \prime} \mid h^{\prime}, \mathbf{r}^{\prime}\right)+\frac{x_{2}}{x_{1}+x_{2}} f_{2}\left(h^{\prime \prime}, \mathbf{r}^{\prime \prime} \mid h, \mathbf{r}\right),
$$

where $x_{1}$ and $x_{2}$ are the magnitudes of the vectors represented by $\left|\mathbf{r}^{\prime \prime}-\mathbf{r}\right|$ and $\left|\mathbf{r}^{\prime \prime}-\mathbf{r}^{\prime}\right|$.

Once the rate of change of the magnitude of $\mathbf{e}$ is obtained by Eq. (13), it can be integrated as shown by Eq. (16), to provide the change in the magnitude of $\mathbf{e}$.

$$
\langle\Delta \mathbf{e}\rangle_{h, h^{\prime}}=\int_{0}^{t}\langle\dot{\mathbf{e}}\rangle_{h, h^{\prime}} \mathrm{d} t
$$

The result of the simulation in the evolution of the probability function can now produce the parameters $c_{i j}$ and $n_{i j}$ in Eq. (11). $\alpha_{i j}$ and $\beta_{i j}$ are only dependent on the volume fractions of the two phases (see Table 1) and, as a result, they do not change. Only the empirical constants $c_{i j}$ and $n_{i j}$ evolve with loading. These constants are determined by fitting the new $r$ values (as the magnitude of $\mathbf{e}$ ) for the same probability functions as in Eq. (11). As a result, a simultaneous set of equations in terms of different $r$ inputs is obtained. The new unknown parameters, $c_{i j}$ and $n_{i j}$, can now be solved from this set of equations.

\section{Simulation and results}

To better understand the behavior of the probability functions during deformation, two examples are chosen for this investigation:

1. a computer generated microstructure, and

2. a $\mathrm{SiC}$ reinforced composite material.

In the case of the aluminum $/ \mathrm{SiC}$ composite material, the microstructures of the inelastically deformed microstructure were scanned and the corresponding probability functions were interpolated. The composite materials were available only for a specific volume fraction. As such, the behavior of the probability functions for different volume fractions could not be investigated. A computer generated 


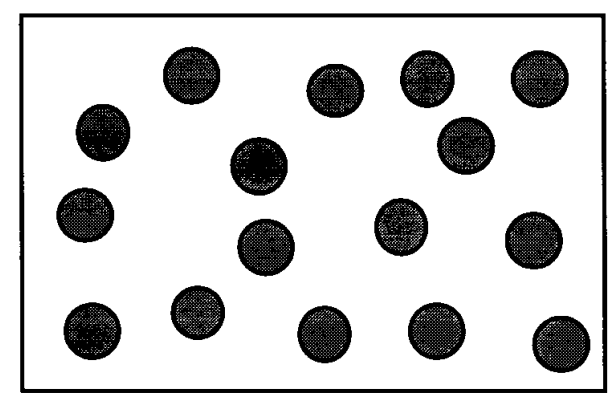

Fig. 2. The microstructure of a two-isotropic-phase medium.

microstructure can be used for such an investigation. In the following, the result of the simulation for the two cases are explained in detail.

\subsection{Computer generated microstructure}

A 2-D composite microstructure (Fig. 2) is generated by allocating centers of circles of radii $r$ at random locations until a specific volume fraction is reached. The theory provided in this paper can be applied only to a 3-D microstructure. The 2-D composite microstructure is then further extended to a 3$\mathrm{D}$ microstructure by assuming that the statistics in the third dimension is the same as the ones for the 2$\mathrm{D}$ composite. This implies that the same initial empirical constants $c_{i j}$ and $n_{i j}$ are used for all directions.

For each phase in a two-isotropic-phase medium, the modulus $N_{i j k l}$ Eq. (2) is derived from a powerlaw steady-state creep (Hutchinson, 1976).

$$
N_{i j k l}=\left[\frac{2}{3} \frac{\sigma^{*}}{D^{*}}\left(\frac{D}{D^{*}}\right)^{m-1}\right] I_{i j k l},
$$

where $D^{*}$ and $\sigma^{*}$ are the strain-rate and stress invariants for the reference configuration, $m$ is the strain rate sensitivity parameter, $D$ is the invariant of the strain-rate tensor and $I_{i j k l}=1 / 2\left(\delta_{i k} \delta_{j l}+\delta_{i l} \delta_{j k}\right)$ is the isotropic fourth rank tensor.

The parameters chosen for the first phase and the second phase in the medium have the relationships with each other as $\sigma_{1}^{*}: \sigma_{2}^{*}=1: 3, n_{1}: n_{2}=1: 2.5$ and $D_{1}^{*}: D_{2}^{*}=1: 1$. Three volume fractions for the second phase are chosen $(0.38,0.58$ and 0.17$)$. Then we obtain three sets of numerical results for the evolution of parameters of the probability function Eq. (11) with increasing strain. A macroscopic strain field is imposed here in the form of uniaxial tension. The final strain along the tension direction is 1.0 (in./in.) and the increment of strain for numerical processing is 0.02 (in./in.).

Fig. 3(a-f) display the results for the study of the evolution of the probability parameters as a function of strain (or during evolution). Because the shape of the second phase is constantly changing, the trend in the evolution of parameters are not exactly the same along different directions. In figures below, for each volume fraction of the second phase, the results of the two perpendicular directions (axial and transverse) are shown for all parameters. This difference in the trend of the evolutionary process results in the expected macroscopic anisotropy of the medium. The reason for such a behavior is that while the second phase is elongated along the tensile direction at higher strains, the average distance between the second phases decreases. Fig. 4 shows the changing trends of macroscopic stresses on the tensile properties for three volume fractions. At constant strain rate and at a low volume fraction 


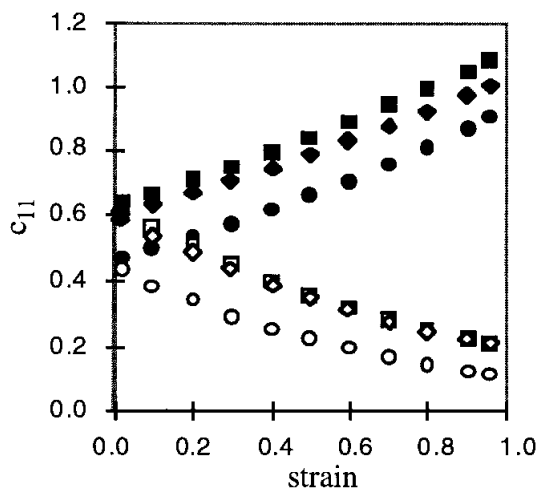

(a)

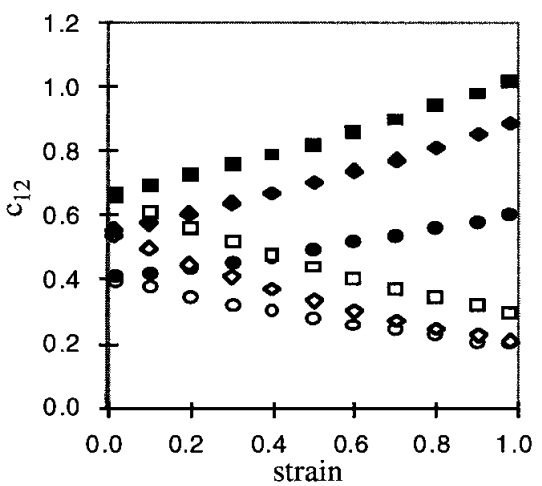

(c)

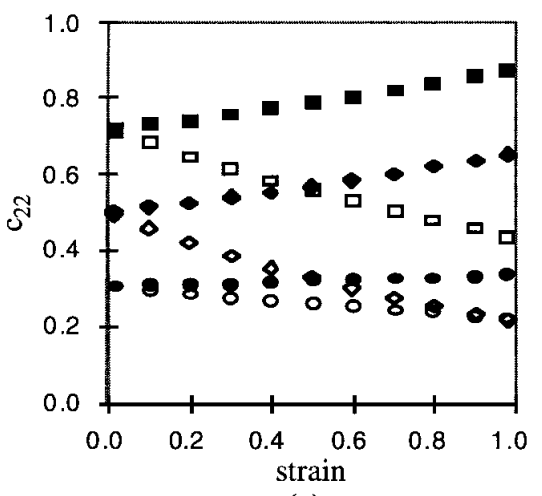

(e)

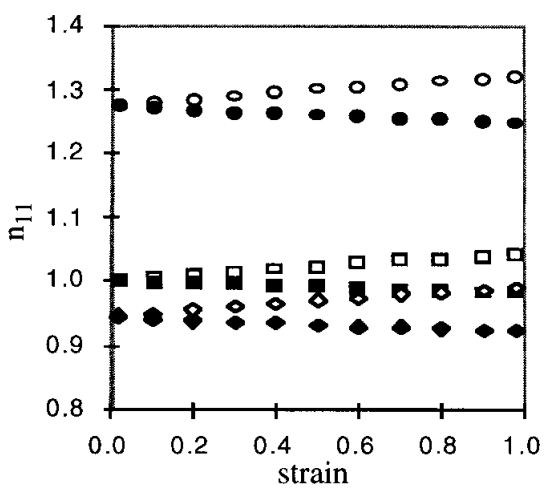

(b)

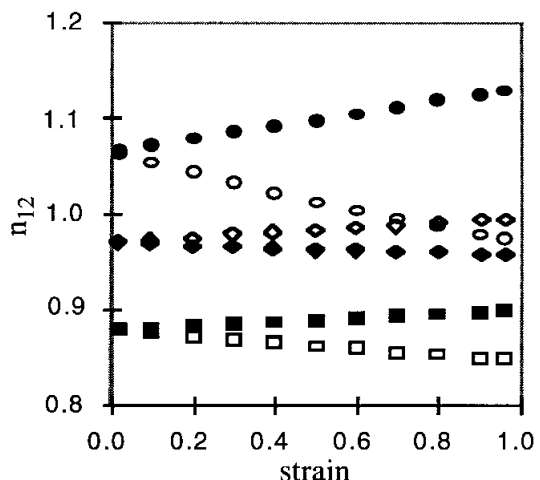

(d)

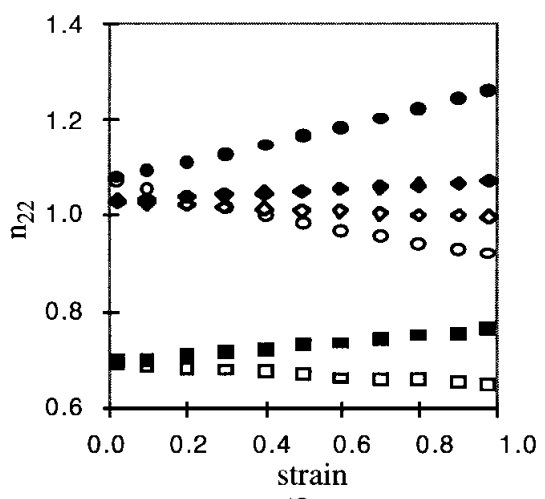

(f)

$0^{0}$ direction $\quad 90^{0}$ direction

$\begin{array}{lll}\mathrm{v}_{2}=0.17 & \circ & \text { - } \\ \mathrm{v}_{2}=0.38 & \text { 口 } \\ \mathrm{v}_{2}=0.58 & \text { ○ }\end{array}$

Fig. 3. (a-f) $c_{i j}$ and $n_{i j}$ evolutions with strains (numerical). 


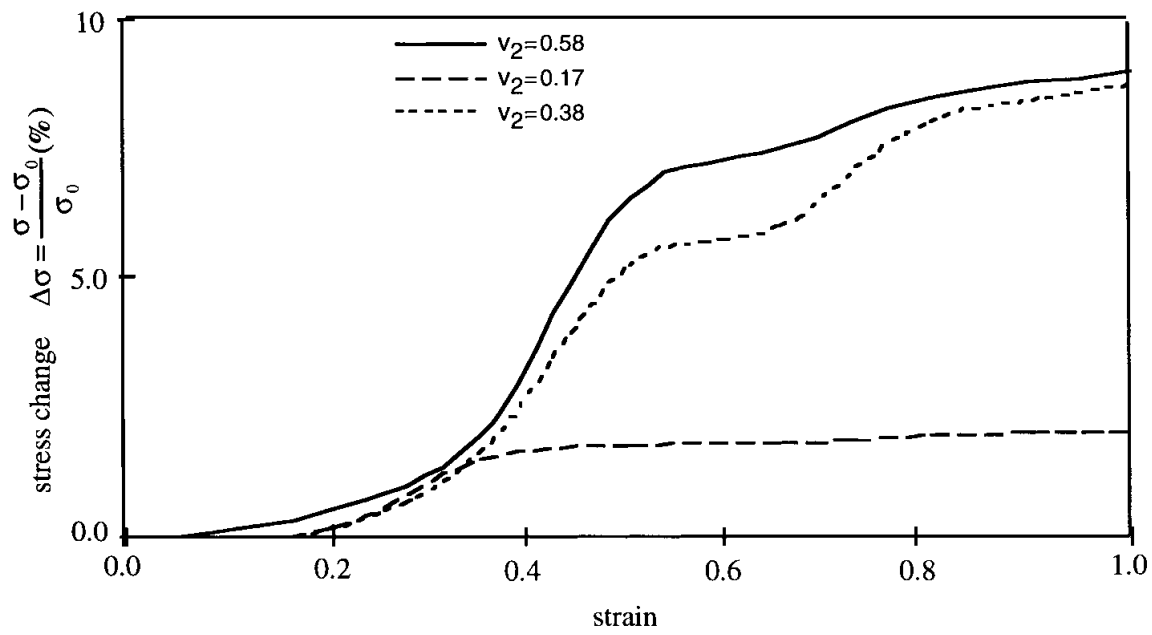

Fig. 4. Microscopic stress change vs. strain.

of the second phase (0.17) the macroscopic effect is trivial because of the negligible shape changes of the second phase. For higher volume fractions, an apparent work hardening behavior is observed.

\subsection{Inelastic deformation of $\mathrm{Al} / \mathrm{SiC}$ composite}

A particulate reinforced composite $(\mathrm{Al} / \mathrm{SiC})$ material is chosen for the purpose of this study. Tensile specimens were machined from this composite and were deformed at a rate of $10^{-4} \mathrm{sec}^{-1}$ and at a temperature of $300^{\circ} \mathrm{C}$. Four specimen were pulled in tension to the strains of $10 \%, 30 \%, 50 \%$ and $100 \%$, respectively. The microstructure of the strained materials was then investigated microscopically and the results are presented in Fig. 5(a-d). The micrographs were digitally scanned and the microstructures were statistically processed to provide the parameters $c_{i j}$ and $n_{i j}$ in Eq. (11). Fig. 6(a-f) shows the evolution of the microstructure as a function of inelastic strain as is measured in the experimentally evaluated parameters $c_{i j}$ and $n_{i j} .0^{\circ}$ and $90^{\circ}$ in the figures represent tension and transverse directions respectively. Comparing with Fig. $3(\mathrm{a}-\mathrm{f})$, it is clear that the experimental results display the same evolutionary behavior with the numerical results. The calculation for $c_{i j}$ shows a general decrease for the $0^{\circ}$ direction and an increase for the $90^{\circ}$ direction. This is in agreement with the simulation. The results for the $n_{i j}$ show an insensitivity for the low levels of strain (up to $30 \%$ ), also in agreement with the simulation. As the strain levels increase, there is a split in the measured $n_{i j}$ values from a more random initial microstructure. For the $n_{11}$ values there is an increase for the $0^{\circ}$ direction and decrease for the $90^{\circ}$ direction. The reverse behavior was measured for the $n_{12}$ and $n_{22}$ results - a decrease for the $0^{\circ}$ direction and increase for the $90^{\circ}$ direction.

\section{Conclusion}

Proper formulation for the evolution of probability function is very essential to the application of the statistical continuum mechanics model to heterogeneous microstructures and their evolution. The result of the investigation performed in this paper presents a clear indication that two point probability functions may be adequate in the representation of the distribution of certain microstructures. The 


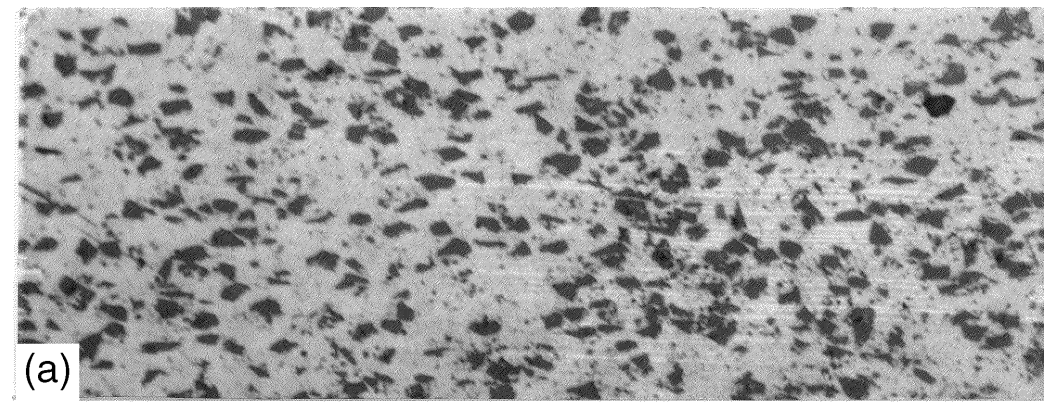

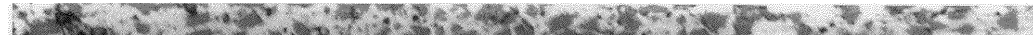

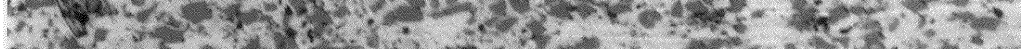

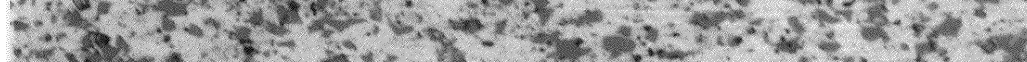

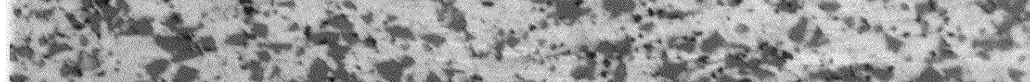

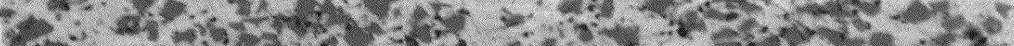

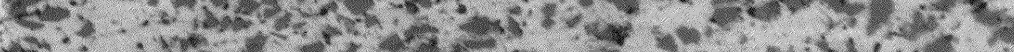

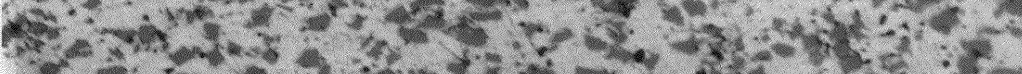

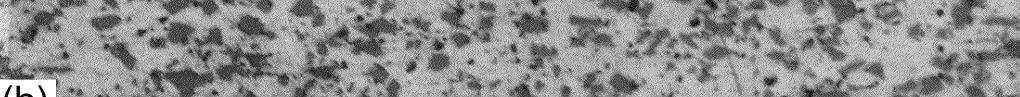

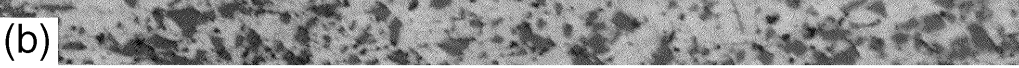
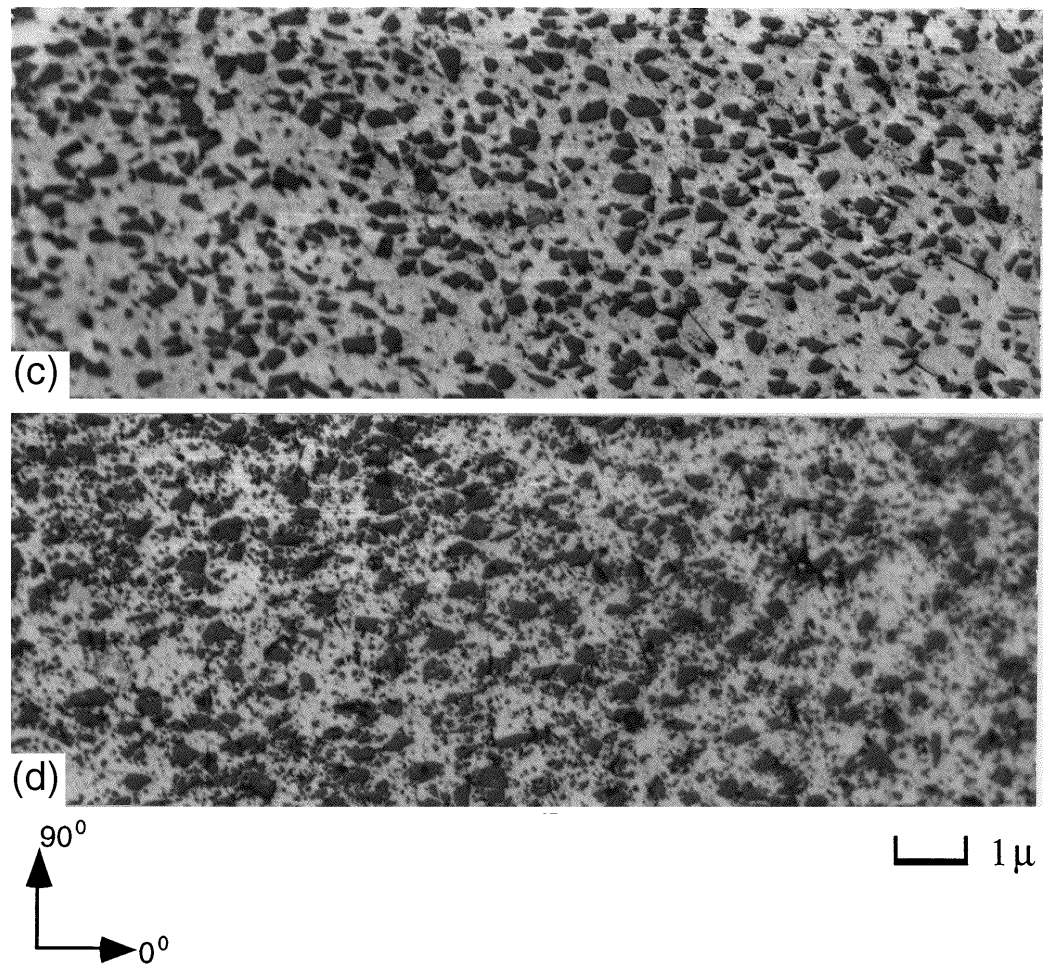

Fig. 5. (a-d) Al-SiC material configurations after 10\%, 30\%, 50\% and $100 \%$ strain. 


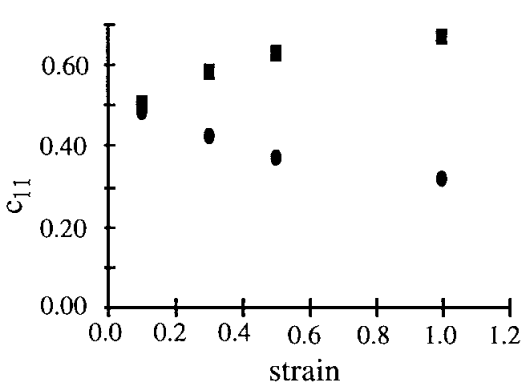

(a)

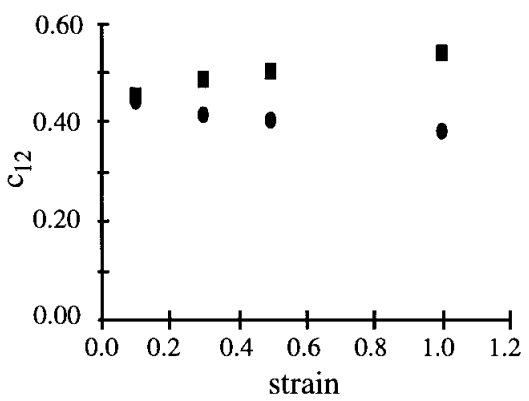

(c)

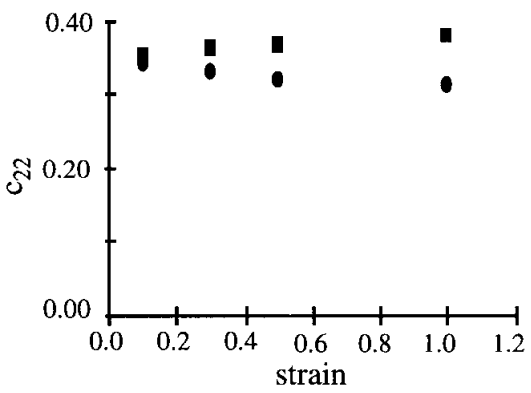

(e)

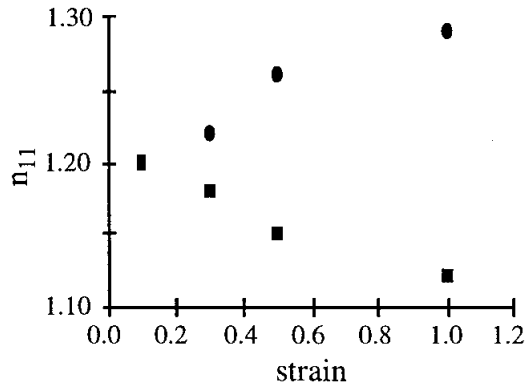

(b)

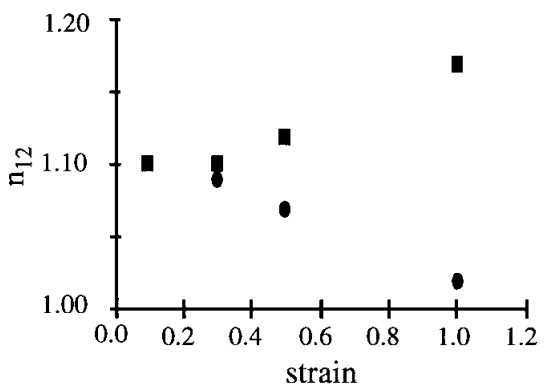

(d)

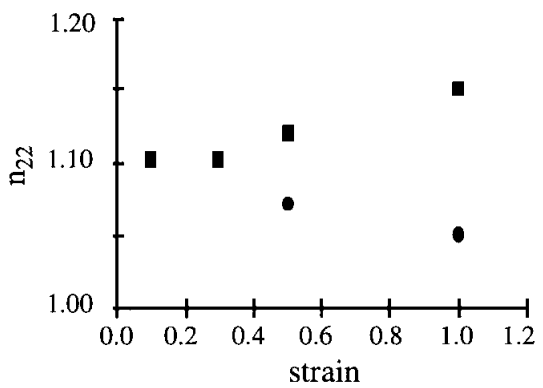

(f)

- $0^{0}$ direction

- $90^{0}$ direction

Fig. 6. (a-f) $c_{i j}$ and $n_{i j}$ evolutions with strains (experimental).

methodology presented here uses a very simplistic formulation for the probability functions and can be thought as an initial attempt. The use of the Secant Modulus and a power law for the inelastic behavior of materials provided a means to investigate the effect of two point probability functions in the evolution of microstructures. The results show that better approximations can be provided to the effective properties of materials using the two point probability functions for some distributions of heterogeneous microstructures. It is however realized that working with the Secant Modulus is not necessarily the best choice. A tangent modulus formulation can soften the global response of the 
material. The evolution of the microstructure resulted in an apparent change (increase) in the effective properties exhibiting an apparent work hardening behavior. A general behavior was discovered for the probability functions for several volume fractions of a computer generated randomly oriented two phase composite. This point was proved experimentally for the case of an $\mathrm{Al} / \mathrm{SiC}$ particulate composite. An orientation dependent probability function would be desirable to represent more complex microstructures and for relatively large-deformations.

\section{References}

Adams, B.L., 1986. Description of the intercrystalline structure distribution in polycrystalline materials. Metall. Trans. 17 A, 2166.

Adams, B.L., Canova, G.R., Molinari, A., 1989. A statistical formulation of viscoplastic behavior in heterogeneous polycrystals. Textures Microstruct. 11, 57-71.

Adams, B.L., Morris, P.B., Wang, T.T., Willden, K.S., Wright, S.I., 1987. Description of orientation coherence in polycrystalline materials. Acta Metall. 35, 2035-2946.

Beran, M.J., 1968. Statistical Continuum Theories. Interscience Publishers, New York.

Beran, M., Molyneux, J., 1966. Use of classical variational principles to determine bounds for the effective bulk modulus in heterogeneous media. Quart. Appl. Math. 24, 107.

Corson, P.B., 1974. Correlation functions for predicting properties of heterogeneous materials. I. Experimental measurement of spatial correlation functions in multiphase solids. J. Applied Physics 45, 3159-3171.

Garmestani, H., Lin, S., Adams, B.L., 1998. Statistical continuum theory for inelastic behavior of a two-phase medium. Int. J. Plasticity 14 (8), 719-731.

Hutchinson, J.W., 1976. Bounds and self-consistent estimates for creep of polycrystalline materials. Proc. Roy. Soc. (London) A $348,101$.

Kröner, E., 1967. Elastic moduli of perfectly disordered composite materials. J. Mech. Phys. Solids 15, 319.

Kröner, E., 1972. Statistical Continuum Mechanics. Springer Verlag, Wien, New York.

Kröner, E., 1977. Bounds for effective elastic moduli of disordered materials. J. Mech. Phys. Solids $25,137$.

Kröner, E., 1987. In: Gittus, J., Zarka, J. (Eds.), Modelling Small Deformation of Polycrystals. Elsevier Applied Science Publishers, New York, p. 229.

Lomakin, V.A., 1965. Deformation of microscopically nonhomogeneous elastic bodies. J. Appl. Math. Mech. 29 , 1048.

Lomakin, V.A., 1970. Statistical Problems of The Mechanics of Deformable Solids. Acad. Sciences, Moscow.

McCoy, J.J., 1981. Nemat Nasser, S. (Ed.), Mechanics Today, vol. 6. Pergamon Press, Oxford, p. 1.

Molinari, A., Canova, G.R., Ahzi, S., 1987. A self consistent approach of the large deformation polycrystal viscoplasticity. Acta Metall. 35, 2983-2994.

Taylor, G.I., 1938. Plastic strain in metals. J. Inst. Metals 62, 307.

Volkov, S.D., Klinskikh, N.A., 1965. Theory of the elastic properties of polycrystals. Physics Metals, Metallogr. 19, 24.

Wang, T.T., Adams, B.L., Morris, P.R., 1990. Development of orientation coherence in plane-strain deformation. Metall. Trans. A21, 2223-2236. 